\title{
Strategy for Reinforcing Drug Knowledge and Accountability in Drug Administration
}

\author{
Carolyn L. Blue, MS, MSN, RN, is Assistant Pro- \\ fessor at Purdue University, School of Nursing, West \\ Lafayette, Indiana.
}

A variety of drugs is being rapidly developed in the pharmacy laboratory by systemic research. Scientific discovery has led to polypharmacy. The resulting numbers of different drugs have made it nearly impossible for nurses to retain knowledge about each specific drug administered. Although studies are limited, reports indicate that the drug knowledge of practicing nurses is not adequate to ensure safety to clients (Markowitz, Pearson, Kay, \& Loewenstein, 1981).

One of the most difficult and complex roles of the clinical nurse is to assess a patient's response to drug therapy and to anticipate potential adverse effects. Jick (1984) and Melmon (1971) reported that adverse drug reactions are, to some extent, preventable. Ideally, the nurse integrates pharmacodynamic principles with steps in the nursing process to ensure optimal monitoring of drug therapy.

Pharmacology is taught as a distinct course in the first year of the baccalaureate nursing program at Purdue University. Although pharmacology is incorporated into nursing courses in the second year, students enrolled in medical-surgical nursing in the third year were having problems applying pharmacodynamics to the clinical setting. An evaluation of nursing students suggested that pharmacology knowledge had not increased significantly beyond the pharmacology course.

Drug cards and reference books having drug information and nursing considera- tions were used by the students and were helpful in providing an overview of drugs administered each week. However, students continued to have difficulty monitoring individual clients for drug effects and relating this role to their nursing practice.

\section{Strategy}

In an attempt to individualize the administration and monitor drug therapy, the students in a medical-surgical clinical course were instructed to generate nursing care plans related to the effects or potential effects of drugs administered to the assigned client. Considering a drug within the context of the nursing process reinforces nursing considerations in pharmacology. Active participation by the students further reinforces the role and importance of monitoring for drug effects.

Student assignments for selected clients are made during an evening clinical session. It is during this three-hour preparatory experience that students assemble drug information and initially assess their assigned client.

The students were instructed to familiarize themselves with the drug name (generic as well as trade names), drug category, mechanism of action, indications, dosage and route, effects, and nursing considerations. Using information about each drug and data gathered from the chart, as well as client interviews and physical assessment, the students are taught to draw conclusions related to pharmacology. The students then determine whether an actual or potential client problem exists and generate nursing diagnoses from a list accepted by the North American Nursing Diagnoses Association. Carpenito's (1987) format is utilized in designing the nursing care plan. This strategy helps students relate drug information to assessment data gathered in actual clinical practice.

In planning care focused on drug administration, students are taught to list client outcomes and interventions to monitor for and reduce adverse effects of drugs. Writing drug-related care plans emphasizes to the clinical nursing student the importance of monitoring drugs. By individualizing nursing interventions, the student learns to incorporate other considerations about the client. This aspect adds meaning to the general nursing considerations and other information from published drug cards and texts.

Students are then instructed to follow their care plans during the morning clinical experience and make evaluations according to client outcomes. Conscientiousness is developed in an effort to monitor and provide care to assess for and reduce adverse effects of drugs administered in the clinical setting.

\section{Student Reaction to the Assignment}

Students expressed initial dissatisfaction with the drug-related nursing care plan. Primarily, they were concerned that their very basic knowledge in pharmacology was not enough to develop nursing diagnoses, much less an entire plan of care. Another concern was that this assignment 


\section{BRIEFS}

would be too time consuming. Some of these initial negative feelings were resolved by limiting nursing diagnoses to two major adverse effects of one or two drugs administered. In addition, the students worked together writing a drugrelated care plan in a preconference session during the first two weeks of the course.

By the end of the semester, nursing students could identify common adverse effects within drug categories and they reported that they were giving more attention to drugs and drug effects. The instructor noted this change when students individualized nursing considerations when verbally talking about any drug regardless of whether a care plan was made.

\section{Summary}

The strategy of including drug-related nursing diagnoses on the nursing care plan can be a helpful tool in fostering drug knowledge and responsibility for nursing care related to drugs. Using the nursing care plan adds meaning to drug cards and other pharmacologic resources. Individualizing drug considerations on the nursing care plan adds another important dimension to nursing practice. Students demonstrated improved proficiency and relevance in applying drug information to client care.

\section{References}

Carpenito, L.G. (1987). Nursing diagnosis: Application to clinical practice. (2nd ed.). Philadelphia: J.B. Lippincott Co.

Jick, H. (1984). Adverse drug reactions: The magnitude of the problem. $J$ Allergy Clin Immunol, 74(4), 555-557.

Markowitz, J.S., Pearson, G., Kay, B.G., \& Loewenstein, R. (1981). Nurses, physicians, and pharmacists: Their knowledge of hazards of medications. Nurs Res, 30(6), 366-370.

Melmon, K.L. (1971). Preventable drug reactions -causes and cures. N Engl J Med, 284, 1361-1368 